08

\title{
Динамические режимы автогенерации сверхвысокочастотного сигнала в радиофотонном кольцевом генераторе
}

\author{
(C) А.В. Кондрашов, А.Б. Устинов, Б.А. Калиникос \\ Санкт-Петербургский государственный электротехнический университет „ЛЭТИ“, \\ 197376 Санкт-Петербург, Россия \\ e-mail: kondrashov_av@inbox.ru
}

(Поступило в Редакцию 28 июня 2016 г.)

Исследованы динамические режимы автогенерации сверхвысокочастотного (СВЧ) сигнала в радиофотонном генераторе. Генератор представлял собой кольцевую схему, в СВЧ тракте которой содержался фильтр нижних частот и СВЧ усилитель. Оптический тракт содержал линию задержки из оптоволокна. Генератор демонстрировал периодическую, хаотическую и шумовую динамики. Показано, что в режиме хаотической генерации корреляционная размерность аттрактора хаотического сигнала приходила в насыщение с ростом размерности фазового пространства. В случае шумовой генерации насыщения не происходило.

DOI: 10.21883/JTF.2017.02.44135.1958

\section{Введение}

В последние годы особый интерес исследователей вызывает разработка сверхвысокочастотных (СВЧ) генераторов динамического хаоса. Он обусловливается возможностью использования хаотических сигналов в телекоммуникационных [1] и радарных [2] системах, а также в генераторах случайных чисел [3]. При этом особое внимание уделяется ширине спектра генерируемого хаотического сигнала. Более широкий спектр позволяет реализовать более высокоскоростную систему связи либо добиться лучшей разрешающей способности радаров.

В литературе описаны СВЧ генераторы широкополосных хаотических сигналов, работающие на различных физических принципах. Так, в работе [4] предложен СВЧ генератор, использующий процесс взаимодействия полупроводниковой сверхрешетки с объемным или микрополосковым резонатором. В работе [5] исследован генератор хаотических колебаний в диапазоне $3-8 \mathrm{GHz}$ на базе биполярного СВЧ транзистора.

В работах [6-9] были предложены электронноперестраиваемые кольцевые генераторы, в которых в качестве нелинейных элементов применялись ферромагнитные пленки и феррит-сегнетоэлектрические структуры. Эти генераторы позволяют управлять параметрами автогенерируемого динамического хаоса с помощью варьирования магнитного или электрического полей, прикладываемых к нелинейному элементу.

В настоящее время интенсивно развивается направление, называемое радиофотоникой [10,11]. Оно объединяет в себе оптоэлектронные и СВЧ технологии, что позволяет сочетать преимущества обеих технологий при разработке новых устройств. Одним из достоинств оптоэлектронных компонентов является их широкополосность. Мгновенная полоса частот у промышленно выпускаемых компонентов достигает $100 \mathrm{GHz}$ и имеет потенциал для дальнейшего расширения. Это делает сравнительно простой возможность создания широкополосных оптоэлектронных генераторов СВЧ динамического хаоса [12].

В недавней работе [13] нами было опубликовано короткое сообщение об экспериментальном исследовании радиофотонного кольцевого генератора широкополосного СВЧ динамического хаоса с равномерной спектральной плотностью мощности в диапазоне 3-8 GHz. Целью настоящей работы являлось детальное исследование динамических режимов, реализующихся при автогенерации СВЧ сигнала в радиофотонном генераторе, а также численная оценка параметров генерируемых СВЧ сигналов.

\section{Экспериментальный макет}

Блок-схема генератора показана на рис. 1. Основными узлами генератора являлись лазер 1 с длиной волны оптического излучения $1.55 \mu \mathrm{m}$ и мощностью $10 \mathrm{~mW}$, электрооптический модулятор типа интерферометра Маха-Цендера 2 с полосой $10 \mathrm{GHz}$ и полуволновым напряжением $3.3 \mathrm{~V}$, оптический волновод 3 диаметром $8 \mu \mathrm{m}$ и длиной $100 \mathrm{~m}$, фотодетектор 4 с верхней рабочей частотой $25 \mathrm{GHz}$, развязка по постоянному току 5, СВЧ усилители 6 с диапазоном частот $2-8 \mathrm{GHz}$, коэффициентом усиления $35 \mathrm{~dB}$ и выходной мощностью в линейном режиме до $1.5 \mathrm{~W}$, переменный аттенюатор 7, СВЧ фильтр нижних частот (ФНЧ) 8 с частотой среза $3.5 \mathrm{GHz}$ и СВЧ направленный ответвитель 9. Компоненты СВЧ тракта соединялись СВЧ кабелями.

Рассмотрим принцип работы генератора. Лазерный диод 1 излучает непрерывное оптическое излучение, выполняющее функцию несущего сигнала. Электрооптический модулятор 2 модулирует амплитуду оптического излучения СВЧ сигналом, поступающим из радиочастотного тракта. Модулированное оптическое излучение, распространяясь по оптоволоконному кабелю 3, задер- 


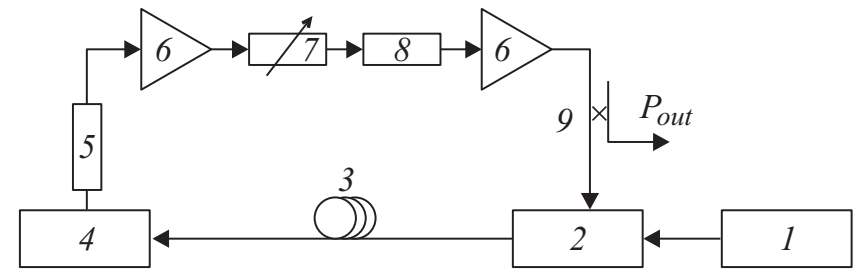

Рис. 1. Блок-схема экспериментального макета.

живается в нем на время около $521 \mathrm{~ns}$ и детектируется фотодетектором 4. Развязка по постоянному току 5 убирает постоянную составляющую напряжения на выходе фотодетектора. Полученный СВЧ сигнал усиливается с помощью СВЧ усилителей 6 и подается на электрооптический модулятор 2, замыкая кольцевую схему. Пара СВЧ усилителей 6 компенсирует суммарные потери на распространение СВЧ сигнала в кольце. Переменный аттенюатор 7 служит для управления этими потерями. Небольшая часть СВЧ сигнала выводится из кольца направленным ответвителем 9 с коэффициентом связи $10 \mathrm{~dB}$

Заметим, что в описанном генераторе частотная полоса хаотической генерации определяется полосой пропускания СВЧ тракта, поэтому последний должен быть широкополосным. В представленной схеме нижнюю частоту хаотической генерации $3.5 \mathrm{GHz}$ задавал ФНЧ 8 . Верхняя частота хаотической генерации определялась верхней граничной частотой СВЧ усилителей и составляла $8 \mathrm{GHz}$.

Нелинейным элементом генератора являлся электрооптический модулятор. Именно косинусоидальная передаточная функция модулятора обусловливала нелинейность преобразования электрического СВЧ сигнала в волну огибающей оптического сигнала. Этот эффект ограничивал в целом СВЧ мощность в режиме генерации. Предварительные тестовые измерения показали, что мощность на выходе СВЧ тракта не превышала $910 \mathrm{~mW}$, поэтому все его узлы работали в линейном режиме.

\section{Экспериментальные результаты}

Экспериментальное исследование перехода к автогенерации хаотического сигнала проводилось путем плавного увеличения значения динамического параметра коэффициента усиления кольца $G$. Увеличение $G$ осуществлялось путем уменьшения ослабления СВЧ сигнала в переменном аттенюаторе 7. Значение $G$, при котором кольцо переходило в режим автогенерации, было условно принято равным нулю. Таким образом, $G=0 \mathrm{~dB}$ соответствовало порогу автогенерации СВЧ сигнала. Увеличение коэффициента усиления кольца приводило к последовательной смене режимов автогенерации, обусловливаемой нелинейным видом переда- точной характеристики электрооптического модулятора. Рассмотрим эти режимы.

На пороге автогенерации в кольце возникала генерация монохроматического СВЧ сигнала. Частота генерируемой гармоники составляла $3.676 \mathrm{GHz}$, а мощность $0.3 \mathrm{~mW}$. Вокруг генерируемой гармоники наблюдались другие частотные пики, являющиеся результатом усиления шума на частотах, соответствующих гармоникам кольца. Они были ослаблены по мощности примерно на 40-50 dB. Поскольку эти гармоники не были связаны по фазе, на выходе генератора наблюдался постоянный уровень СВЧ мощности.

При увеличении коэффициента усиления $G$ выше $7.3 \mathrm{~dB}$ мощность СВЧ сигнала на входе электрооптического модулятора начинала превышать $55 \mathrm{~mW}$. Выше этой мощности в кольце происходила бифуркация, в результате которой возникала бистабильность. Одночастотная генерация сменялась многочастотной, причем центральная частота генерации перескакивала на гармонику с частотой $3.652 \mathrm{GHz}$. В спектре СВЧ сигнала при этом возникали гармоники с двумя частотами отстройки, примерно равными 1.92 и $178.6 \mathrm{MHz}$. В таком режиме наблюдались быстрые осцилляции различной длительности, которые далее мы будем называть цугами. Период быстрых осцилляций $T_{1}$ был около $5.6 \mathrm{~ns}$. Длительность цугов варьировалась и повторялась через некоторое количество цугов. В последовательности цугов можно было выделить некоторый период $T_{2}=520.8 \mathrm{~ns}$ (назовем его периодом медленных колебаний), который практически не зависел от $G$. Таким образом, каждый период медленных колебаний содержал несколько цугов быстрых осцилляций.

Описанный режим генерации являлся периодическим, поскольку соотношения частот генерируемых гармоник были кратными, а во временной области наблюдался амплитудно модулированный СВЧ сигнал с периодом $T_{2}$ (рис. 2 и 3). Длительность и форма цугов на периоде менялась при увеличении коэффициента усиления кольца. Рассмотрим подробнее то, как выглядит сигнал во времени в таком режиме генерации.

На рис. 2, а показана временная реализация при $G=9 \mathrm{~dB}$. Видно, что на каждом периоде медленных колебаний $T_{2}$ наблюдались три цуга длительностью 30 , 90 и 120 ns. При $G=9.2 \mathrm{~dB}$ (рис. $2, b$ ) длительность цугов составила 20,50 и $250 \mathrm{~ns}$. На рис. $3, a, b$ показана в разных масштабах временная реализация, измеренная для $G=9.4 \mathrm{~dB}$. При таком $G$ на периоде $T_{2}$ вместо нескольких цугов наблюдался один, состоявший из большого числа быстрых осцилляций. Таким образом, при достижении $G=9.4 \mathrm{~dB}$ все три цуга сливались в один, длительность которого была равна $450 \mathrm{~ns}$. Быстрые осцилляции имели форму периодической последовательности темных нелинейных импульсов (рис. $3, b$ ). Период импульсов составлял $5.49 \mathrm{~ns}$, а их длительность была около $1.90 \mathrm{~ns}$. На рис. 3, $c$, $d$ показан спектр такого сигнала в разных масштабах. Видно, что спектр состоял из гармоник с большой и маленькой отстройкой по 

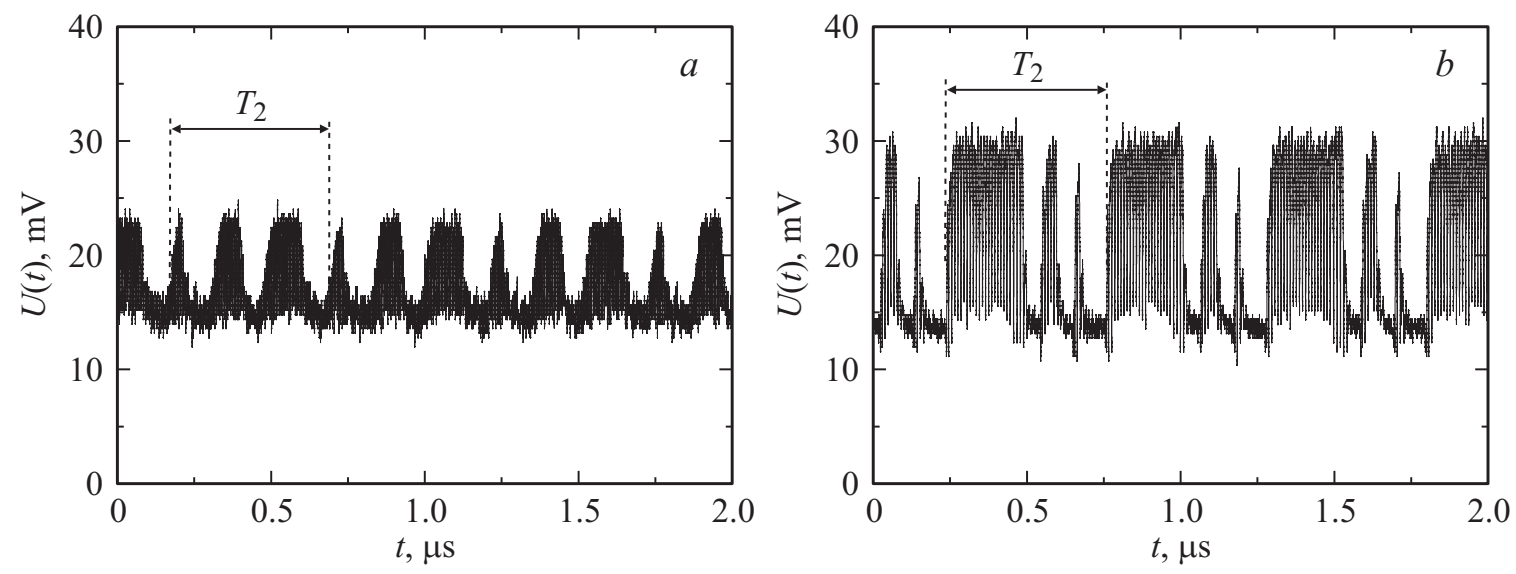

Рис. 2. Осциллограммы СВЧ сигналов, генерируемых при $G=9 \mathrm{~dB}: a-9, b-9.2$.
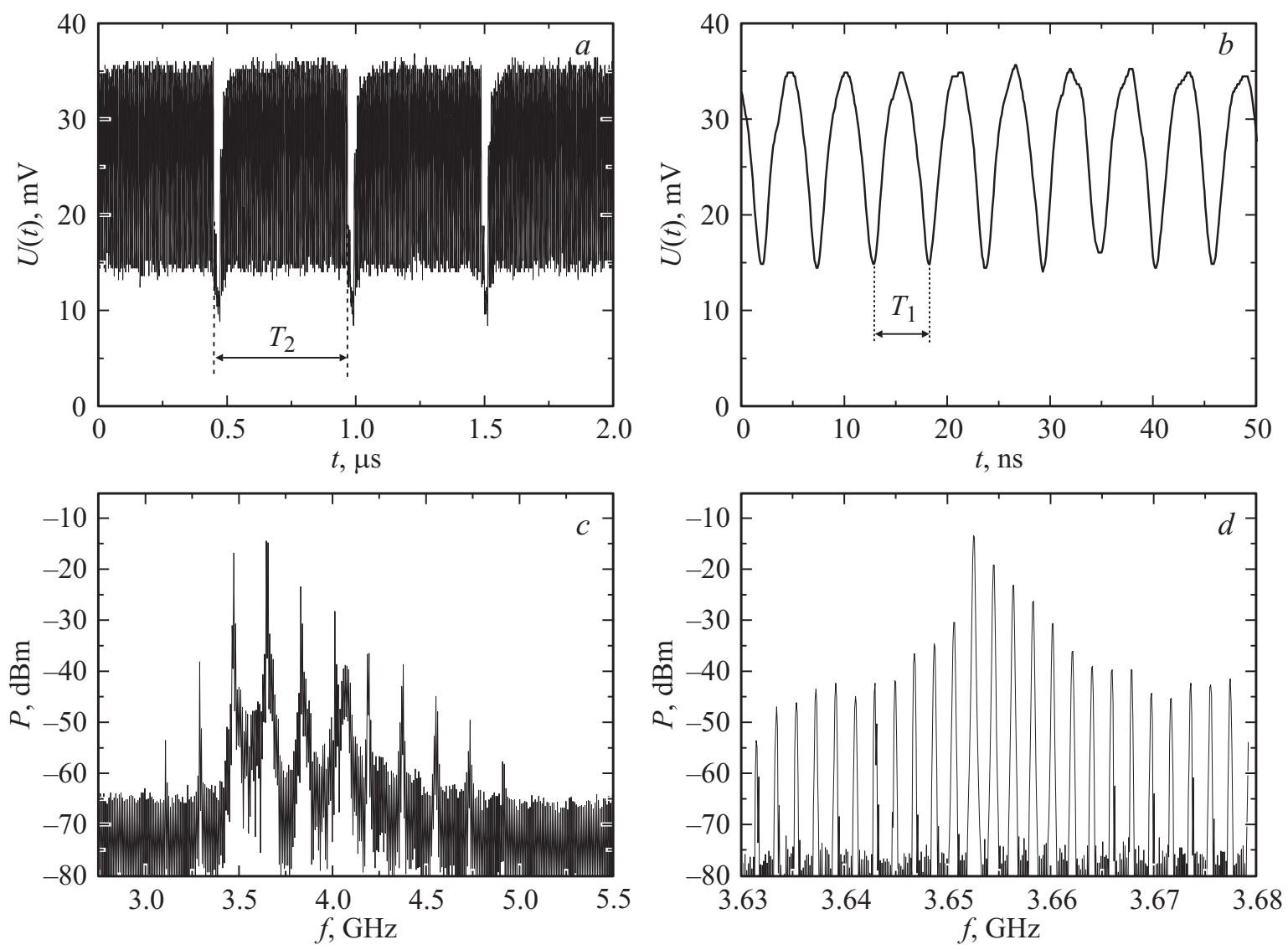

Рис. 3. Осциллограмма СВЧ сигнала при $G=9.4 \mathrm{~dB}$, показывающая медленные осцилляции амплитуды (a), и та же осциллограмма в расширенном масштабе, показывающая быстрые осцилляции амплитуды $(b)$, а также спектр СВЧ сигнала $(c)$ и его фрагмент в расширенном масштабе $(d)$.

частоте, определявших форму, период и длительность медленных и быстрых осцилляций. Эти отстройки были равны 182.4 и $1.92 \mathrm{MHz}$ соответственно.

При дальнейшем увеличении коэффициента усиления $G$ выше $11.7 \mathrm{~dB}$ колебания в кольце стохастизировались, и спектр становился сплошным. На левой части рис. 4 представлены спектр и фрагмент осциллограммы хаотического сигнала, измеренные при $G=15.1 \mathrm{~dB}$. Из рисунка можно видеть, что спектр выходного СВЧ сигнала представлял собой сплошной шумовой пьедестал с отдельными ярко выраженными гармониками. Эти гармоники соответствовали частотам собственных мод кольца. Огибающая генерируемого СВЧ сигнала представляла собой последовательность хорошо различимых 

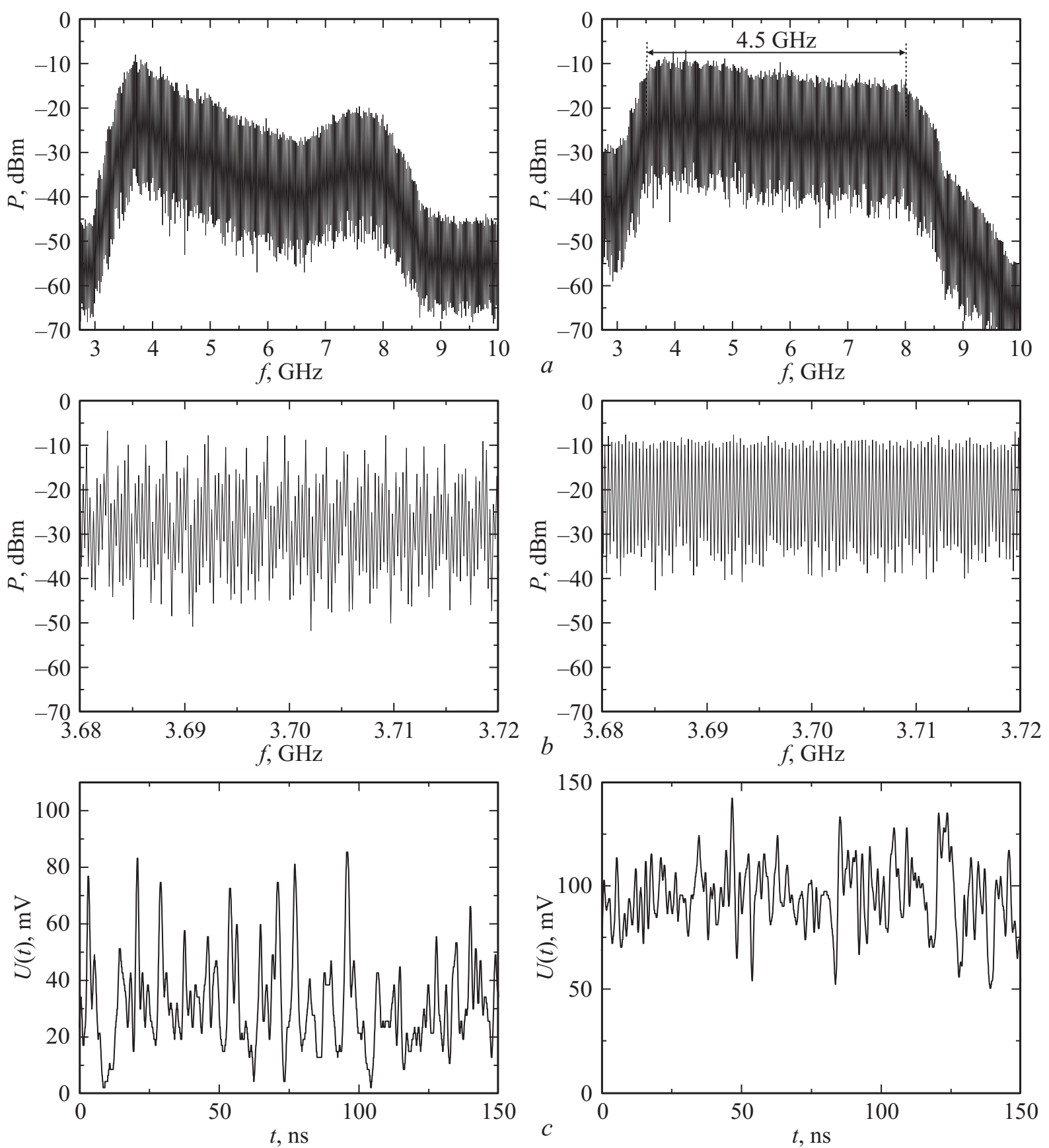

Рис. 4. Спектры $(a)$, их фрагменты в расширенном масштабе $(b)$ и фрагменты осциллограмм $(c)$, измеренные при хаотической генерации для $G=15.1 \mathrm{~dB}$ (слева) и при шумовой генерации для $G=22.9 \mathrm{~dB}$ (справа).

хаотических импульсов. Стохастизация колебаний в исследуемой кольцевой системе объясняется амплитудным механизмом перехода к динамическому хаосу, который обычно наблюдается в кольцевых автоколебательных системах, содержащих нелинейный элемент, передаточная характеристика которого имеет спадающий участок $[14,15]$.

При достижении $G$ значения $18.7 \mathrm{~dB}$ происходила смена режима генерации, при которой отдельные гармоники пропадали. Спектр выходного СВЧ сигнала становился сплошным, а сигнал во времени приобретал сложную апериодическую форму. Спектральная характеристика и временная реализация, записанные для $G=22.9 \mathrm{~dB}$, показаны на правых панелях рис. 4.

\section{Анализ режимов генерации}

Из анализа временных реализаций было определено, является ли генерируемый непериодический сигнал квазипериодическим сигналом, динамическим хаосом или 
случайным шумом [16]. Для анализа режимов генерации записывались осциллограммы длительностью $10 \mu \mathrm{s} \mathrm{c}$ шагом между точками 100 ps. На первом этапе производилось построение фазовых портретов наблюдавшихся режимов генерации методом задержки. Координаты точек фазовых траекторий получались путем сдвига временной реализации на некоторое время задержки в соответствии с выражением

$$
\mathbf{x}(t)=\{U(t), U(t+\tau), \ldots, U(t+(d-1) \tau)\},
$$

где $U(t)$ - огибающая измеренного сигнала, $\tau$ - время задержки, $d-$ размерность фазового пространства.

На рис. 5, $a$ представлен фазовый портрет колебаний в периодическим режиме генерации для $G=9.4 \mathrm{~dB}$, временная реализация которого показана на рис. 3. Фазовый портрет представляет собой замкнутую петлю. Форма петли определялась формой быстрых осцилляций. В промежутки времени, в течение которых наблюдались быстрые осцилляции, динамика системы заключалась в движении по этой замкнутой петле. В промежутки времени между осцилляциями система перескакивала в точку, соответствующую низкому уровню генерируемой мощности. Для $G=9.4 \mathrm{~dB}$ эта точка имела координаты $(10 \mathrm{mV}, 10 \mathrm{mV})$. Перескоки носили периодический характер. Для значений $G$, при которых реализовывался этот режим работы генератора, фазовый портрет был аналогичным. Увеличение коэффициета усиления увеличивало время движения системы по замкнутой петле и уменьшало время нахождения в точке.

Вследствие стохастизации колебаний при $G>11.7 \mathrm{~dB}$ форма аттрактора значительно изменялась. Траектории начинали занимать в фазовом пространстве некоторый объем, плотно заполняя его. На рис. 5, $b$ показан фазовый портрет, соответствующий временной реализации при $G=15.1 \mathrm{~dB}$, представленной на левой части рис. 4,c. Поскольку в этом режиме наблюдались хаотические импульсы с основанием вблизи уровня $0 \mathrm{mV}$, фазовый портрет был вытянут к началу координат. Бифуркация, происходящая при $G=18.7 \mathrm{~dB}$, изменяла временную реализацию, что приводило к изменению фазового портрета. Фазовые траектории начинали вращаться вокруг некоторой точки фазового пространства, не приближаясь к началу координат (рис. 5, c).

Бесструктурные фазовые портреты, показанные на рис. $5 b, c$, характерны для систем, генерирующих либо хаотический сигнал с большой фрактальной размерностью, либо сигнал, имеющий природу случайного шума, фрактальная размерность которого не ограничена [16]. Чтобы выяснить природу генерируемых сигналов, был проведен расчет фрактальных размерностей полученных аттракторов.

Для расчета значений фрактальной размерности был использован алгоритм Грассбергера-Прокаччиа [17]. Он заключается в покрытии всего фазового портрета гиперсферами с характеристическим размером $l$ и построении зависимости величины корреляционной суммы $C$ от значения $l$. Под корреляционной суммой $C$ понимается среднее число точек фазового портрета, попадающих внутрь сферы. Значения корреляционной суммы и характеристического расстояния связаны соотношением

$$
C(l) \propto l^{D_{C}},
$$

где $D_{C}-$ корреляционная размерность проекции аттрактора в $d$-мерное фазовое пространство. Ее величина характеризует геометрическую форму проекции аттрактора, т.е. его топологию. Значение корреляционной размерности может меняться при увеличении размерности фазового пространства $d$, что объясняется более полным совпадением топологии аттрактора с топологией его проекции. Если начиная с некоторого значения $d$ корреляционная размерность $D_{C}$ проекции перестает изменяться, то топология проекции совпадает с топологией аттрактора, а полученное значение размерности $D_{C}$ проекции является фрактальной размерностью аттрактора.

На рис. 6, $a$ в логарифмическом масштабе показаны графики зависимостей корреляционной суммы от характеристического расстояния для аттрактора, полученного при $G=15.1 \mathrm{~dB}$ (рис. 5,b). В таком масштабе наклон линейной области зависимостей соответствует значению $D_{C}$. Разные линии на графике соответствуют различным значениям размерности фазового пространства $d$.

В соответствии с вышеизложенным можно видеть, что с ростом $d$ наклон линейных областей зависимостей $\lg (C)$ от $\lg (l)$ сначала возрастает, что соответствует росту $D_{C}$. Затем при достижении $d=28$ наклон перестает заметно изменяться. Это говорит о том, что дальнейшее увеличение размерности фазового пространства не приводит к более точному определению топологии фазового портрета, а его размерность равна размерности странного аттрактора.

На рис. 6,b показаны графики зависимостей $\lg (C)$ от $\lg (l)$ для аттрактора, полученного при $G=22.9 \mathrm{~dB}$ (рис. 5,c). Из рисунка видно, что наклон линейных областей зависимостей растет вплоть до достаточно большого значения $d=50$, не демонстрируя замедления роста. Это говорит об отсутствии упорядоченности во взаимном расположении траекторий фазового портрета.

На основе результатов, показанных на рис. 6, были построены зависимости корреляционных размерностей $D_{C}$ от размерности фазового пространства $d$, которые приведены на рис. 7. Из рисунка видно, что для $G=15.1 \mathrm{~dB}$ зависимость $D_{C}(d)$ выходит на уровень 11.5 и далее не изменяется при размерности фазового пространства $d=28$. Наличие уровня насыщения говорит о том, что при $G=15.1 \mathrm{~dB}$ форма фазового портрета и его проекций определялись формой странного аттрактора, возникавшего в фазовом пространстве. Фрактальная размерность аттрактора равнялась 11.5 , а минимальная размерность вложения равнялась 28. Конечные значения фрактальной и минимальной размерностей вложения 

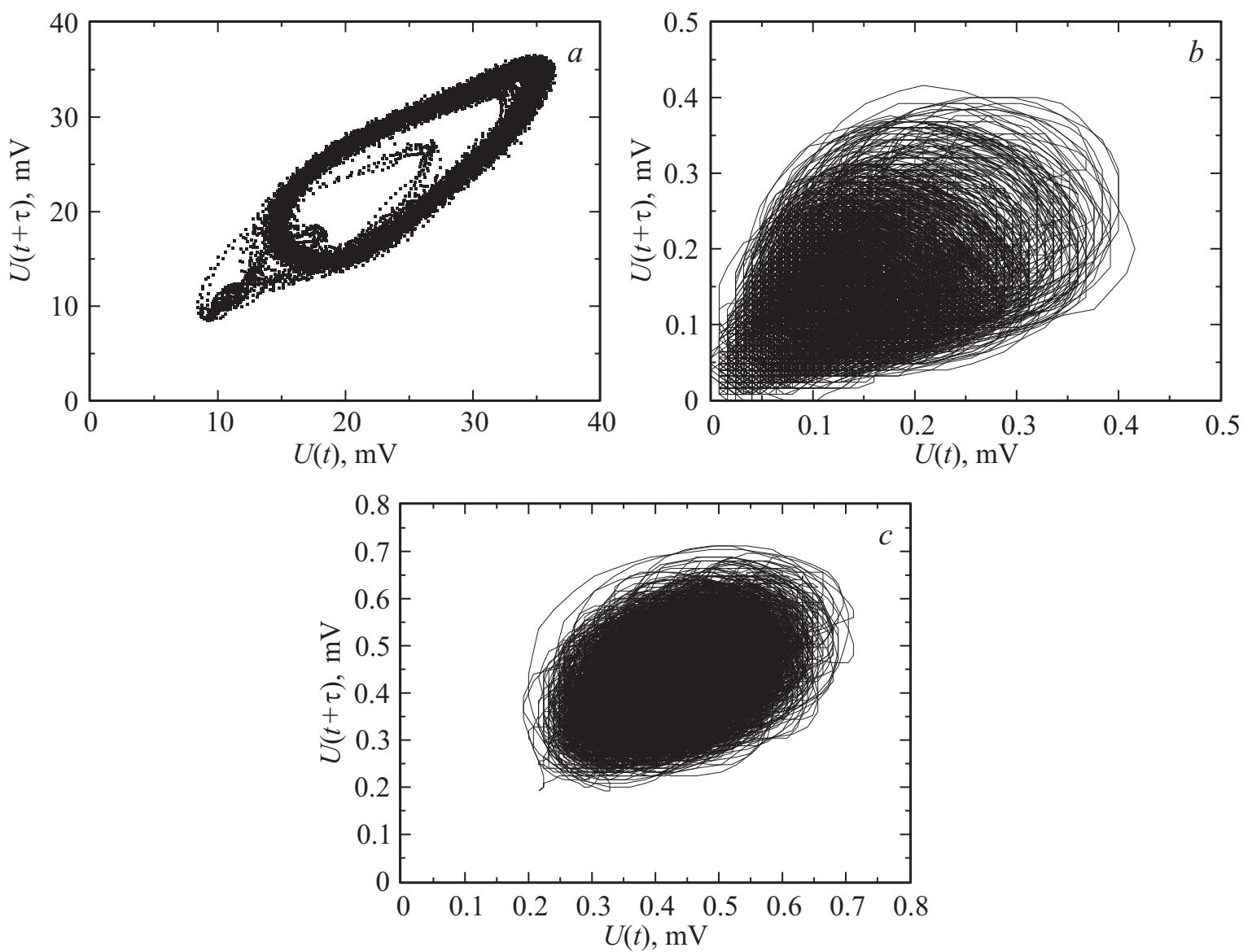

Рис. 5. Двумерные фазовые портреты СВЧ сигнала, генерируемого при $G=9.4 \mathrm{~dB}(a)$, хаотической генерации для $G=15.1 \mathrm{~dB}(b)$ и при шумовой генерации для $G=22.9 \mathrm{~dB}(c)$.
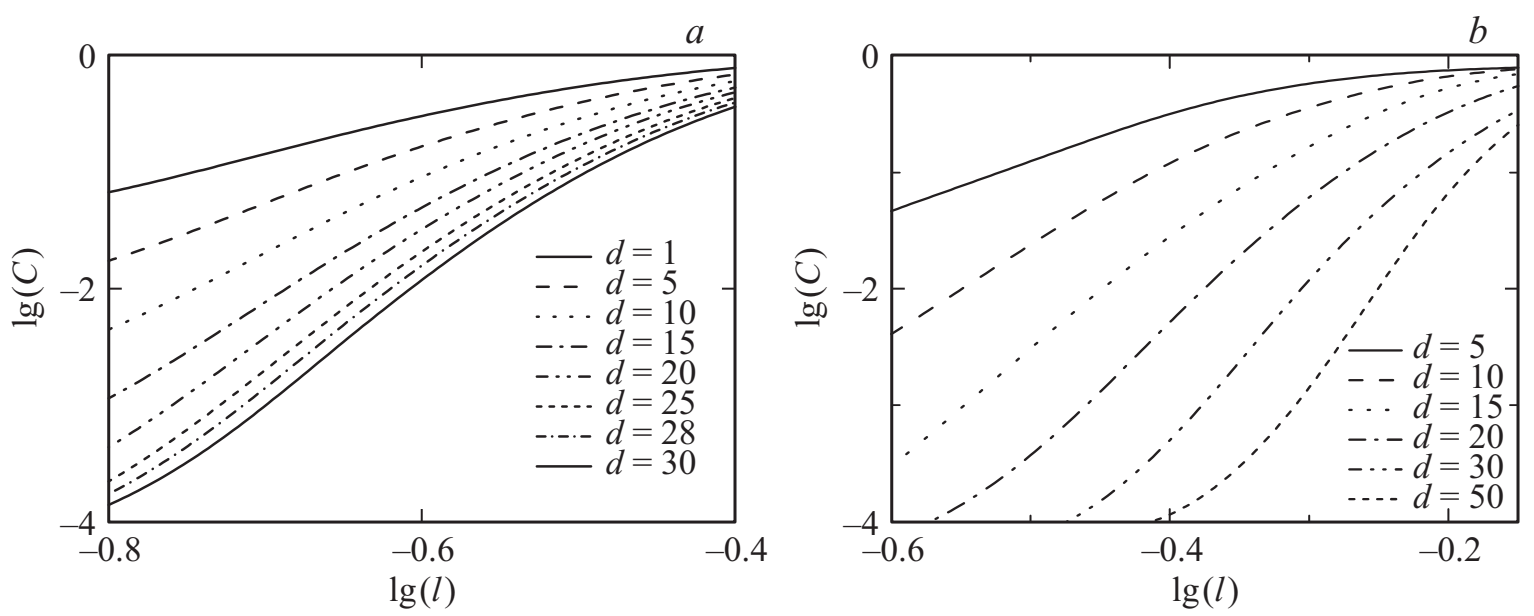

Рис. 6. Зависимости корреляционного интеграла от характеристического расстояния в логарифмическом масштабе, соответствующие хаотической генерации для $G=15.1 \mathrm{~dB}(a)$ и при шумовой генерации для $G=22.9 \mathrm{~dB}(b)$.

характеризуют наблюдавшийся сигнал как динамический хаос [16]. Зависимость $D_{C}(d)$ для $G=22.9 \mathrm{~dB}$ не имеет уровня насыщения, что свидетельствует в пользу шумовой природы генерируемого сигнала. Таким образом, результаты анализа фазовых портретов позволяют за- ключить, что при $G=18.7 \mathrm{~dB}$ генерация динамического хаоса в радиофотонном генераторе сменялась шумовой генерацией.

Помимо значения фрактальной размерности были рассчитаны значения старших показателей Ляпунова, 


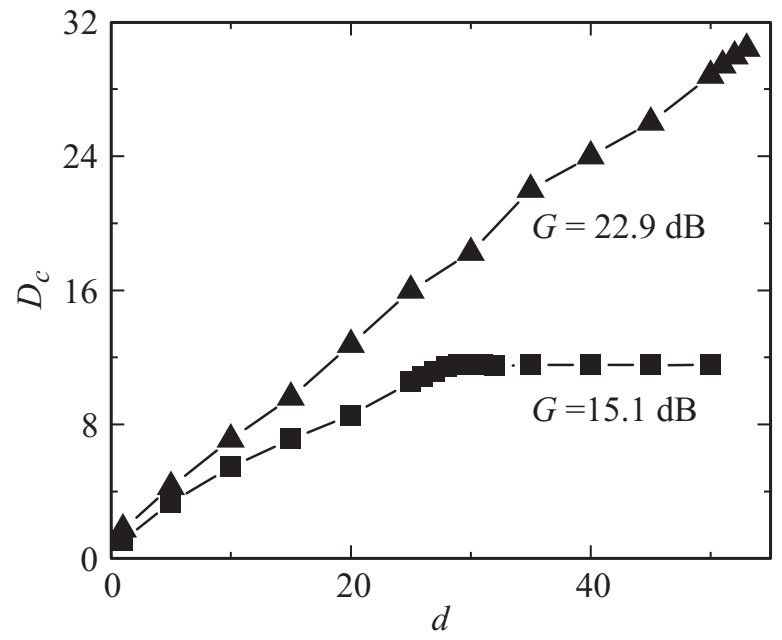

Рис. 7. Зависимости корреляционной размерности от размерности вложения, соответствующие хаотической генерации для $G=15.1 \mathrm{~dB}$ (квадратики) и шумовой генерации для $G=22.9 \mathrm{~dB}$ (треугольники).

характеризующих устойчивость аттрактора в фазовом пространстве. Расчет был проведен для размерности фазового пространства $d=28$ с помощью программного пакета Tisean [18]. Расчеты показали, что при $G=15.1 \mathrm{~dB}$ старший Ляпуновский показатель имел значение $2.8 \mu \mathrm{s}^{-1}$, а при $G=22.9 \mathrm{~dB}$ он равнялся $14.8 \mu \mathrm{s}^{-1}$. Полученные значения указывают на то, что в обоих случаях существует перемешивание фазовых траекторий в фазовом пространстве, но во втором случае разбегание траекторий фазового пространства более интенсивное. Это подтверждает то, что в радиофотонном генераторе с увеличением коэффициента усиления кольца сначала происходит переход от регулярной динамики к хаотической, а затем к шумовой.

\section{Заключение}

Проведенное исследование показывает, что радиофотонный кольцевой генератор демонстрирует богатую нелинейную динамику. Для периодических режимов автогенерации характерны колебания с большой и малой частотами отстройки. При хаотической генерации для спектра характерны повышенный шумовой пьедестал с отдельными ярко выраженными гармониками, а временные реализации имеют вид хаотических последовательностей импульсов. В режиме шумовой генерации спектр сигнала имеет равномерную плотность мощности, а сигнал во времени имеет сложную апериодическую форму.

Вид динамического режима, реализующегося в генераторе, подтверждается результатами анализа осциллограмм. В режиме хаотической генерации корреляционная размерность с ростом размерности вложения выходит в насыщение. При шумовой генерации такого насыщения не происходит. Сценарий перехода от авто- генерации монохроматического сигнала к хаотической, а затем к шумовой автогенерации не носит универсальный характер.

Экспериментальная часть работы выполнена при частичной поддержке грантов РФФИ № 14-02-00496 и 15-32-20357 мол_а_вед, гранта президента Российской Федерации для государственной поддержки ведущих научных школ, а также госзадания Минобрнауки РФ. Теоретическая часть работы выполнена при поддержке Российского научного фонда (грант № 16-12-10440).

\section{Список литературы}

[1] Chaotic Signals in Digital Communications / Ed. by M. Eisencraft, R. Attux, R. Suyama. Boca Raton: CRC Press, 2013. 264 p.

[2] Chaotic Signal Processing / Ed. by H. Leung. Philadelphia: SIAM, 2013. 367 p.

[3] Li W., Reidler I., Aviad Y., Huang Y., Song H., Zhang Y., Rosenbluh M., Kante I. // Phys. Rev. Lett. 2013. Vol. 111. P. 044102.

[4] Hramov A.E., Makarov V.V., Koronovskii A.A., Kurkin S.A., Gaifullin M.B., Alexeeva N.V., Alekseev K.N., Greenaway M.T., Fromhold T.M., Patané A., Kusmartsev F.V., Maksimenko V.A., Moskalenko O.I., Balanov A.G. // Phys. Rev. Lett. 2014. Vol. 112. P. 116603.

[5] Дмитриев А.С., Ефремова Е.В., Румянцев Н.В. // Письма в ЖТФ. 2014. Т. 40. Вып. 2. С. 1-9.

[6] Wu M., Kalinikos B.A., Patton C.E. // Phys. Rev. Lett. 2005. Vol. 95. P. 237202.

[7] Гришин С.В., Гришин В.С., Романенко Д.В., Шараевский Ю.П. // Письма в ЖТФ. 2014. Т. 40. Вып. 19. C. 51-59.

[8] Ustinov A.B., Kondrashov A.V., Nikitin A.A., Kalinikos B.A. // Appl. Phys. Lett. 2014. Vol. 104. P. 234101.

[9] Hagerstrom A., Wu M., Eykholt R., Kalinikos B.A. // Phys. Rev. B. 2011. Vol. 83. P. 104402.

[10] Белкин М.Е., Сигов А.С. // Радиотехн. и электрон. 2009. T. 54. Вып. 8. С. 901-914.

[11] Liang W., Eliyahu D., Ilchenko V.S., Savchenkov A.A., Matsko A.B., Seidel D., Maleki L. // Nat. Commun. 2015. Vol. 6. P. 7957.

[12] Callan K.E., Illing L., Gao Z., Gauthier D.J., Schöll E. // Phys. Rev. Lett. 2010. Vol. 104. P. 113901.

[13] Устинов А.Б., Кондрашов А.В., Калиникос Б.А. // Письма в ЖТФ. 2016. Т. 42. В. 8. С. $28-36$.

[14] Залогин Н.Н., Кислов В.В. Широкополосные хаотические сигналы в радио-технических и информационных системах. М.: Радиотехника, 2006. 208 с.

[15] Бегинин Е.Н., Гришин С.В., Шараевский Ю.П. // Письма в ЖТФ. 2010. Т. 36. Вып. 7. С. 65-74.

[16] Шредер М. Фракталы, хаос, степенные законы. Миниатюры из бесконечного рая. Ижевск: НИЦ „Регулярная и хаотическая динамика“, 2001. 528 с.

[17] Grassberger P., Procaccia I. // Phys. Rev. Lett. 1983. Vol. 50 P. 346-348.

[18] Schreiber T., Schmitz A. // Phys. D. 2000. Vol. 142. P. 346-350. 